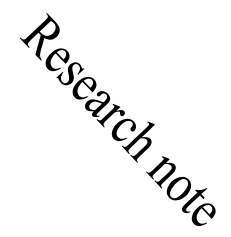

\title{
Tunable surface polaritons of one-dimensional photonic crystal containing graphen-based hyperbolic metamaterials
}

\author{
A Madani, Re Abdi-Ghaleh, J Poursamad \\ Department of Photonics, Laser and Optical Engineering, University of Bonab, Bonab, Iran \\ E-mail: a-madani@bonabu.ac.ir
}

(Received 12 February 2019 ; in final form 14 September 2019)

\begin{abstract}
In this paper, surface polaritons (SPs) at the interface of a semi-infinite homogeneous dielectric medium and a onedimensional photonic crystal (PC) have been investigated theoretically. The PC is made of the alternate layers of an isotropic ordinary dielectric and a graphene-based hyperbolic metamaterial layer. The effective medium approach has been used to study the metamaterial layers, showing that they have hyperbolic dispersion in a certain frequency range at the $\mathrm{THz}$ region. The obtained results show that the structure has some photonic band gaps in the hyperbolic and elliptical frequency regions for both TE and TM polarizations and can support the SPs in these frequency ranges. It is observed that the characteristics of the SPs depend on the geometrical parameters of the structure and the optical parameters of the graphene monolayers. In the following, the electromagnetic field profiles of some SPs have been plotted, showing that the modes of the first band gap are more localized than those of the second one. Finally, the intensity distribution of a TM-polarized Gaussian beam inside and outside the PC has been simulated, verifying the localization of the SPs at the interface of the structure.
\end{abstract}

Keywords: surface polaritons, graphene-based hyperbolic metamaterial, photonic crystal, tunable

For full article, refer to the Persian section 